\title{
Axionlike Lee-Wick particles in light-shining-through-wall experiments
}

\author{
Christopher D. Carone* \\ High Energy Theory Group, Department of Physics, William \& Mary, Williamsburg, \\ Virginia 23187-8795, USA
}

(Received 29 October 2020; accepted 15 December 2020; published 30 December 2020)

\begin{abstract}
We consider the possibility of an axionlike particle (ALP) that is a ghost, with wrong-sign kinetic and mass terms. Such an ALP can arise as the partner to an ordinary particle in theories with higher-derivative quadratic terms. We compute the photon regeneration probability in light-shining-through-wall experiments and show that the presence of such a ghostly ALP can lead, in principle, to discernible effects.
\end{abstract}

DOI: 10.1103/PhysRevD.102.115039

\section{INTRODUCTION}

The axion is the pseudo-Goldstone boson of a spontaneously broken $\mathrm{U}(1)$ global symmetry that is anomalous with respect to the $\mathrm{SU}(3)$ color gauge group of the standard model [1]. Since models with axions can solve the strong $C P$ problem [2], axion phenomenology has been the focus of considerable attention. Axionlike particles (ALPs) have a similar low-energy effective Lagrangian and can be probed via the same experimental techniques used to search for axions [3]. Whether or not an ALP contributes to the solution of the strong $C P$ problem, the fact that such particles can arise so generically in extensions of the standard model makes them of interest for phenomenological investigation.

In the case where the ALP is pseudoscalar, its coupling to photons is given by the Lagrangian

$\mathcal{L}=-\frac{1}{4} F_{\mu \nu} F^{\mu \nu}+\frac{1}{2} \partial_{\mu} a \partial^{\mu} a-\frac{1}{2} m^{2} a^{2}+\frac{1}{4} g_{a \gamma \gamma} a \tilde{F}_{\mu \nu} F^{\mu \nu}$,

where $F_{\mu \nu}$ is the photon field strength tensor, $\tilde{F}^{\mu \nu} \equiv$ $\frac{1}{2} \epsilon^{\mu \nu \alpha \beta} F_{\alpha \beta}$ is its dual, and $g_{a \gamma \gamma}$ is a dimensionful coupling, with units of inverse mass. In the case where the ALP is a scalar, we may relabel $a \rightarrow s$ and replace the interaction term in Eq. (1.1) with $\frac{1}{4} g_{s \gamma \gamma} s F_{\mu \nu} F^{\mu \nu}$. It has been long known that the ALP-photon interaction may lead to the conversion of photons to ALPs in the presence of a strong magnetic field. While photons from an incident beam can be blocked by a barrier, the ALPs may proceed through it and be

\footnotetext{
*dcaro@wm.edu
}

Published by the American Physical Society under the terms of the Creative Commons Attribution 4.0 International license. Further distribution of this work must maintain attribution to the author(s) and the published article's title, journal citation, and DOI. Funded by SCOAP. converted back to photons subsequently in the presence of a magnetic field on the other side [4]. Such light-shiningthrough-wall (LSW) experiments have provided excluded regions in the $m_{A^{-}} g_{A \gamma \gamma}$ plane [5], for $A=a$ or $s$.

The possibility we consider here is a second ALP that appears in a higher-derivative generalization of this effective Lagrangian, with quadratic terms

$$
\mathcal{L}=-\frac{1}{2} \hat{a} \square \hat{a}-\frac{1}{2} m^{2} \hat{a}^{2}-\frac{1}{2 M^{2}} \hat{a} \square^{2} \hat{a} .
$$

In cases where the distinction between scalar and pseudoscalar does not matter, we will refer to the ALP as $a$, with a hat to signify the higher-derivative form of the theory. In a theory where the ALP is associated with the spontaneous breaking of a global symmetry via the vacuum expectation value of a complex scalar field $\phi$, a similar higherderivative term for $\phi$ in its Lagrangian leads to Eq. (1.2), as we will see later. In the next section, we review how Eq. (1.2) can be recast as a theory without higher-derivative terms, but with a new field $\tilde{a}$ that has wrong-sign kinetic and mass terms, i.e., one that corresponds to a Lee-Wick ghost [6]. Like the Lee-Wick standard model [7], such a theory will have a unitary $S$ matrix provided that (i) the Lee-Wick partner is excluded from the spectrum of asymptotic states [6] and (ii) certain prescriptions are applied in evaluating loop diagrams [8]. If one assumes that Lee-Wick partners serve as a solution to the hierarchy problem, then partners must be present for every particle, including in sectors that represent extensions of the standard model. The masses of these partner particles are free parameters; no physical consideration forces a common Lee-Wick scale for all the partner particles, though this is often assumed in the literature as a simplifying assumption. We will therefore take $M$ as a free parameter in our discussion. We will see that the coupling of $\tilde{a}$ to photons (and in fact to anything else) will have the same form as an ordinary ALP, justifying the choice to call it an ALP as well. 
In this paper, we investigate the effects of a light LeeWick ALP on LSW experiments. The most common computation of the probability for regeneration of photons on the far side of a barrier first computes the probability of a photon converting to an ALP (and vice versa) in a magnetic field, as inferred from solutions to classical field equations (see, for example, Ref. [4]). This approach is not useful for taking into account the virtual Lee-Wick contribution or its interference with the ordinary one; we instead approach the problem in a quantum field theory setting, maintaining the requirement that the Lee-Wick partner not appear in a Feynman diagram as an external line. We show that the new contribution to the scattering amplitude alters the photon regeneration probability when both the ordinary and LeeWick ALPs are light. The effect we identify is one that might be experimentally discerned. It is worth noting that there are many other examples in the literature of extensions of the standard model, such as supersymmetry, in which the possibility of a light member of the spectrum of new particles, such as a light gluino [9] or light axino [10], has been investigated due to their interesting phenomenological implications; the present work is similarly motivated and the unusual nature of the ghostlike particles that we consider makes our study distinct.

In the next section we discuss the relevant effective theory, and in Sec. III present the computation of the probability relevant to LSW experiments. In the final section, we summarize our conclusions.

\section{LEE-WICK ALPS}

The procedure for converting the Lagrangian in Eq. (1.2) into one without the higher-derivative term, by use of an auxiliary field, is now well known from the literature on the Lee-Wick standard model [7]. Here we give only a brief review.

The following Lagrangian:

$\mathcal{L}_{A F}=-\frac{1}{2} \hat{a} \square \hat{a}-\frac{1}{2} m^{2} \hat{a}^{2}-\tilde{a} \square \hat{a}+\frac{1}{2} M^{2} \tilde{a}^{2}+\mathcal{L}_{\text {int }}(\hat{a})$

can be shown to be equivalent to Eq. (1.2) by functionally integrating over the auxiliary field $\tilde{a}$ in the generating functional for the theory. Rewriting this in terms of the shifted field

$$
\hat{a}=a-\tilde{a},
$$

the Lagrangian becomes

$$
\begin{aligned}
\mathcal{L}= & -\frac{1}{2} a \square a+\frac{1}{2} \tilde{a} \square \tilde{a}-\frac{1}{2} m^{2}(a-\tilde{a})^{2}+\frac{1}{2} M^{2} \tilde{a}^{2} \\
& +\mathcal{L}_{\text {int }}(a-\tilde{a}) .
\end{aligned}
$$

In the case where $m=0$, it is clear by inspection that $\tilde{a}$ corresponds to a particle of mass $M$ with wrong-sign kinetic and mass terms. In the case where $m \neq 0$, there is mass mixing. Since the kinetic terms are already diagonal, with opposite signs corresponding to the propagator residues found in the higher-derivative form of the theory, one can only diagonalize the mass matrix using a transformation that preserve the kinetic term matrix $\operatorname{diag}(1,-1)$. Applying this constraint to the components of an arbitrary, real two-by-two transformation matrix leaves one degree of freedom, which can be parameterized

$$
\left(\begin{array}{l}
a \\
\tilde{a}
\end{array}\right)=\left(\begin{array}{cc}
\cosh \theta & \sinh \theta \\
\sinh \theta & \cosh \theta
\end{array}\right)\left(\begin{array}{c}
a_{0} \\
\tilde{a}_{0}
\end{array}\right),
$$

for transformations that are continuously connected to the identity. [This is a symplectic transformation [11], since Eq. (2.4) also leaves a two-by-two nondegenerate skewsymmetric matrix invariant.] This leads to the form

$$
\begin{aligned}
\mathcal{L}= & -\frac{1}{2} a_{0} \square a_{0}+\frac{1}{2} \tilde{a}_{0} \square \tilde{a}_{0}-\frac{1}{2} m_{0}^{2} a_{0}^{2}+\frac{1}{2} M_{0}^{2} \tilde{a}_{0}^{2} \\
& +\mathcal{L}_{\text {int }}\left(e^{-\theta}\left[a_{0}-\tilde{a}_{0}\right]\right),
\end{aligned}
$$

where the 0 subscript indicates a mass eigenstate. One can treat either $(m, M)$ or $\left(m_{0}, M_{0}\right)$ as free parameters. We will do the latter, in which case the mixing angle $\theta$ is determined by ${ }^{1}$

$$
\tanh \theta=-\frac{m_{0}^{2}}{M_{0}^{2}}
$$

The effect of field redefinition that we have described implies that the coupling of $a_{0}$ and $\tilde{a}_{0}$ to photons is given by

$$
\mathcal{L}_{\text {int }} \supset \frac{1}{4} g_{a \gamma \gamma} e^{-\theta}\left(a_{0}-\tilde{a}_{0}\right) \tilde{F}_{\mu \nu} F^{\mu \nu},
$$

where the same substitutions described earlier may be applied in the case where the ALPs are scalar rather than pseudoscalar. Equation (2.7) will be used in the calculation of Sec. III.

The wrong-sign quadratic terms of $\tilde{a}_{0}$ in Eq. (2.5) have consequences for unitarity and causality, issues that have been discussed extensively in the past literature on LeeWick theories $[6,8,13]$. Lee and Wick [6] were first to point out that unitarity can be preserved in such a theory provided that the Lee-Wick partners are excluded from the spectrum of asymptotic scattering states. Cutkosky et al. [8] developed a prescription for the appropriate evaluation of loop diagrams, specifying the deformation of contours around poles in the complex plane so that unitarity is preserved

\footnotetext{
${ }^{1}$ Equation (2.6) is equivalent to $\tanh 2 \theta=-2 m_{0}^{2} M_{0}^{2} /\left(m_{0}^{4}+M_{0}^{4}\right)$ found in Ref. [12].
} 
beyond tree level. In a more modern treatment, Grinstein, O'Connell, and Wise [13] confirmed unitarity in a scalar $\mathrm{O}(\mathrm{N})$ model, showing the importance of contributions from multiparticle states in assuring agreement between the leftand right-hand sides of the optical theorem. They also study another feature of such theories, the apparent violation of microcausality in the wrong-sign vertex ordering for the production and decay of a Lee-Wick resonance, an effect determined indirectly from the asymptotic positions and momenta of incoming and scattered wave-packet states. The significance of the demonstrated unitarity of Lee-Wick theories is that it implies that the microscopic violation of causality can lead to no logical paradoxes, as the unitary $S$ matrix provides a one-to-one mapping from past to future descriptions of the system [13]. Hence, Refs. [6,8,13] suggest that the framework for the ALP that we have adopted is one that is free of any inconsistencies.

When the ALP arises via the spontaneous breaking of an approximate global symmetry, it is often identified via a nonlinear redefinition of a complex scalar field that provides the symmetry-breaking vacuum expectation value $f / \sqrt{2}$ :

$$
\hat{\phi}=\frac{1}{\sqrt{2}}(\hat{\rho}+f) e^{i \hat{a} / f} .
$$

In this case, we assume the Lagrangian

$\mathcal{L}=-\hat{\phi}^{*} \square \hat{\phi}-\frac{1}{M^{2}} \hat{\phi}^{*} \square^{2} \hat{\phi}+m_{\phi}^{2} \hat{\phi}^{*} \hat{\phi}-\frac{\lambda}{2}\left(\hat{\phi}^{*} \hat{\phi}\right)^{2}$,

which includes a higher-derivative quadratic term and provides for the spontaneous breaking of the U(1) global symmetry when $m_{\phi}^{2}>0$. Substituting Eq. (2.8) into Eq. (2.9), one obtains an effective Lagrangian for $\hat{a}$ and $\hat{\rho}$ of the form

$$
\begin{aligned}
\mathcal{L}= & -\frac{1}{2} \hat{a} \square \hat{a}-\frac{1}{2 M^{2}} \hat{a} \square^{2} \hat{a}-\frac{1}{2} \hat{\rho} \square \hat{\rho}-\frac{1}{2 M^{2}} \hat{\rho} \square^{2} \hat{\rho} \\
& -\left[-\frac{1}{2} m_{\phi}^{2}(\hat{\rho}+f)^{2}+\frac{\lambda}{8}(\hat{\rho}+f)^{4}\right]+\mathcal{L}_{\text {int }},
\end{aligned}
$$

where $\mathcal{L}_{\text {int }}$ include derivative interactions involving $\hat{\rho}$ and $\hat{a}$, as well as couplings to standard model fields that may be arise, for example, via loops of heavy particles. The potential for $\hat{\rho}$ is minimized for $f=\sqrt{2 / \lambda} m_{\phi}$, and one finds that $m_{\rho}^{2}=2 m_{\phi}^{2}$. In axion models, the scale of $f$, and hence the mass scale for $\hat{\rho}$, is extremely high; even if it were as low as a TeV in a generic ALP model, this would place the mass scale for $\hat{\rho}$ far above the sub-meV scale of relevance to LSW experiments. Thus, we may work in an effective theory in which $\hat{\rho}$, or equivalently $\rho$ and its LeeWick partner $\tilde{\rho}$, are integrated out of the theory. We are left with

$$
\begin{aligned}
\mathcal{L}= & -\frac{1}{2} \hat{a} \square \hat{a}-\frac{1}{2 M^{2}} \hat{a} \square^{2} \hat{a}-\frac{1}{2 M^{2} f^{2}} \partial^{\mu} \hat{a} \partial^{\nu} \hat{a} \partial_{\mu} \hat{a} \partial_{\nu} \hat{a} \\
& +\frac{1}{4} g_{a \gamma \gamma} \hat{a} \tilde{F}_{\mu \nu} F^{\mu \nu}+\cdots,
\end{aligned}
$$

where the ellipsis refer to any other induced couplings to standard model fields. The point here is that the higherderivative term that we assumed previously for $\hat{a}$ is present; had we included a small, explicit breaking of the U(1) global symmetry in Eq. (2.9), we would also generate the $\hat{a}$ mass term of Eq. (1.2) as well. The only new term is the higher-derivative interaction that is quartic in $\hat{a}$. It is interesting to note that this term is not suppressed by four powers of the high scale $f$ but by $f^{2} M^{2}$ instead, due to the fact that it originates from application of the nonlinear field redefinition to the $1 / M^{2}$-suppressed higher-derivative term in Eq. (2.9). This term can lead to a three-body decay of $\tilde{a}$, though decays may also arise via nonderivative interactions that appear when explicit breaking of the U(1) symmetry is taken into account. Whether this term can have any other phenomenological consequences will not have any relevance to the calculation that we present in the next section.

\section{LIGHT SHINING THROUGH WALLS}

To start, consider a toy example of the interaction between two real scalar fields $\chi$ and $\psi$ with classical sources, $V(x)_{L}$ and $V(x)_{R}$, via the interaction Hamiltonian

$$
H_{\int}=\int d^{4} x\left[\chi \psi V(x)_{L}+\chi \psi V(x)_{R}\right]
$$

Let us assume that $\psi$ is massless and consider one-into-one scattering of $\psi$ particles, in the presence of these sources. In addition, we assume that the interaction region includes a barrier that is impenetrable to $\psi$ particles; the sources $V_{L}$ $\left(V_{R}\right)$ are assumed to be nonvanishing over a finite region to the left (right) of the barrier. As the asymptotic states include only $\psi$ particles, and no intermediate measurement is made of the $\chi$ particles, the quantum mechanical scattering amplitude is given by

$$
\begin{aligned}
i \mathcal{M}= & -\int d^{4} x \int d^{4} x^{\prime} D_{F}\left(x-x^{\prime}\right) V(x)_{L} V\left(x^{\prime}\right)_{R} \\
& \times\left\langle p^{\prime}\left|\psi(x) \psi\left(x^{\prime}\right)\right| p\right\rangle,
\end{aligned}
$$

where $D_{F}\left(x-x^{\prime}\right)$ is the Feynman propagator for the $\chi$ field. After a series of elementary manipulations, this can be reduced to the momentum space expression

$i \mathcal{M}=\int \frac{d^{4} q}{(2 \pi)^{4}} \frac{i}{q^{2}-m_{\chi}^{2}+i \epsilon}\left[i \tilde{V}\left(p^{\prime}-q\right)_{L}\right]\left[i \tilde{V}(q-p)_{R}\right]$,

where $\tilde{V}$ is the Fourier transform of a given source (following the conventions of Peskin and Schroeder [14]) 
and $p\left(p^{\prime}\right)$ represents the four-momentum of the incoming (outgoing) $\psi$ particle. The arguments of $\tilde{V}$ represent the momenta flowing out of each source; conservation of momentum at the vertices leaves one momentum, $q$, unconstrained, which is integrated over in the amplitude.

The analogous approach may be applied to the ALPphoton vertex that we encountered previously, with combinatoric factors included to take into account that there are two ways to choose which field strength tensor in each vertex is treated as the classical source. Assume for the moment that the Lee-Wick ALP is absent (which is useful for comparing our result to the one obtained by the classical approach in Ref. [4]). The amplitude that is analogous to Eq. (3.3) is

$$
\begin{aligned}
i \mathcal{M}= & \frac{g_{a \gamma \gamma}^{2}}{4} \epsilon^{\mu \nu \alpha \beta} \epsilon^{\rho \sigma \kappa \eta} \epsilon_{\mu}(p) \epsilon_{\rho}\left(p^{\prime}\right)^{*} p_{\nu} p_{\sigma}^{\prime} \\
& \times \int \frac{d^{4} q}{(2 \pi)^{4}} \frac{i}{q^{2}-m_{0}^{2}+i \epsilon}\left[i \tilde{F}_{\alpha \beta}^{c l}(q-p)_{L}\right] \\
& \times\left[i \tilde{F}_{\kappa \eta}^{c l}\left(p^{\prime}-q\right)_{R}\right],
\end{aligned}
$$

where the $\epsilon(p)$ and $\epsilon\left(p^{\prime}\right)^{*}$ four-vectors encode the photon polarizations. The tildes in Eq. (3.4) indicate a Fourier transform, not a dual tensor; the latter is taken into account via the Levi-Civita symbols in the prefactor. We now work in the laboratory frame, where we assume a fixed magnetic field $\vec{B}$ in the $z$ direction, and consider polarized photons moving in the $x$ direction, with their electric fields aligned with the applied $B$ field, namely,

$$
F^{\mu \nu}=\left(\begin{array}{cccc}
0 & 0 & 0 & 0 \\
0 & 0 & -B_{z} & 0 \\
0 & B_{z} & 0 & 0 \\
0 & 0 & 0 & 0
\end{array}\right), \quad p^{\mu}=\left(\begin{array}{l}
\omega \\
\omega \\
0 \\
0
\end{array}\right), \quad \epsilon^{\mu}=\left(\begin{array}{l}
0 \\
0 \\
0 \\
1
\end{array}\right) .
$$

To proceed, it is simplest to substitute these choices into Eq. (3.4), square the amplitude, and sum over the final photon polarization states, yielding

$$
|\mathcal{M}|^{2}=g_{a \gamma \gamma}^{4} \omega^{2}\left(p_{0}^{\prime 2}-p_{3}^{\prime 2}\right)\left|I_{0}\left(p^{\prime}, p\right)\right|^{2},
$$

where

$$
I_{0}\left(p^{\prime}, p\right)=\int \frac{d^{4} q}{(2 \pi)^{4}} \frac{\tilde{B}_{z}^{(L)}(q-p) \tilde{B}_{z}^{(R)}\left(p^{\prime}-q\right)}{q^{2}-m_{0}^{2}+i \epsilon} .
$$

We assume that the magnetic field is constant, with magnitude $B_{0}$, and significantly greater in spatial extent than the incoming photon beam in the $y$ and $z$ directions. In the $x$ direction, the magnetic field is constant over the finite interval $-L / 2 \leq x \leq L / 2$ (to the left of the wall) and
$L / 2 \leq x \leq 3 L / 2$ (to the right of the wall) and vanishing otherwise; the wall is assumed to be thin and located at $x=L / 2$. With these choices, the Fourier transform of the magnetic field in the $x$ direction

$$
\int d x B_{z}^{(L)}(x) e^{-i q x}=B_{0} L\left[\frac{\sin (q L / 2)}{q L / 2}\right] \equiv B_{0} L F(q),
$$

where the Fourier transforms in the 0,2, and 3 directions yield delta functions. Here and henceforth, we use $q$ to represent one-dimensional momentum in the $x$ direction, rather than a four-momentum. For the source on the right of the wall,

$$
\begin{aligned}
& \int d x B_{z}^{(R)}(x) e^{-i q x} \\
& =\int d x B_{z}^{(L)}(x-L) e^{-i k q}=B_{0} L e^{-i q L} F(q) .
\end{aligned}
$$

Combining Eqs. (3.7)-(3.9), one may write the squared amplitude as

$$
|\mathcal{M}|^{2}=g_{\text {ary }}^{4} \omega^{4} B_{0}^{4} L^{4}\left[(2 \pi)^{3} \delta\left(p^{\prime 0}-\omega\right) \delta\left(p^{\prime 2}\right) \delta\left(p^{\prime 3}\right)\right]^{2}|I(\omega)|^{2},
$$

where $I(\omega)$ is the one-dimensional integral

$$
I(\omega)=\int \frac{d q}{2 \pi} \frac{F(q-\omega)^{2} e^{i(q-\omega) L}}{q^{2}-\left(\omega^{2}-m_{0}^{2}\right)-i \epsilon} .
$$

We note that the quantity in brackets can be written as $\left[(2 \pi)^{3} \delta^{(3)}(0)\right] \delta\left(p^{\prime 0}-\omega\right) \delta\left(p^{\prime 2}\right) \delta\left(p^{\prime 3}\right)$, while the scattering probability is determined by

$$
\begin{aligned}
d P & =\frac{1}{\langle i \mid i\rangle} \frac{d^{3} p^{\prime}}{(2 \pi)^{3}} \frac{1}{2 \omega^{\prime}}|\mathcal{M}|^{2} \\
& \equiv \frac{1}{2 \omega\left[(2 \pi)^{3} \delta^{(3)}(0)\right]} \frac{d^{3} p^{\prime}}{(2 \pi)^{3}} \frac{1}{2 \omega^{\prime}}|\mathcal{M}|^{2} .
\end{aligned}
$$

The prefactor takes into account the normalization of the incoming scattering states. The divergent factor cancels (or, more rigorously, can be canceled after regulating the delta functions by making the four-volume of the Universe finite), while the remaining three delta functions are eliminated by the phase space integration. We are left with the scattering probability

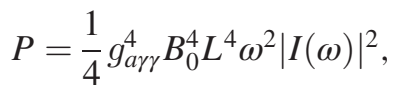

with $I(\omega)$ as given previously. We now evaluate that integral: For convenience, we first shift the integration variable 

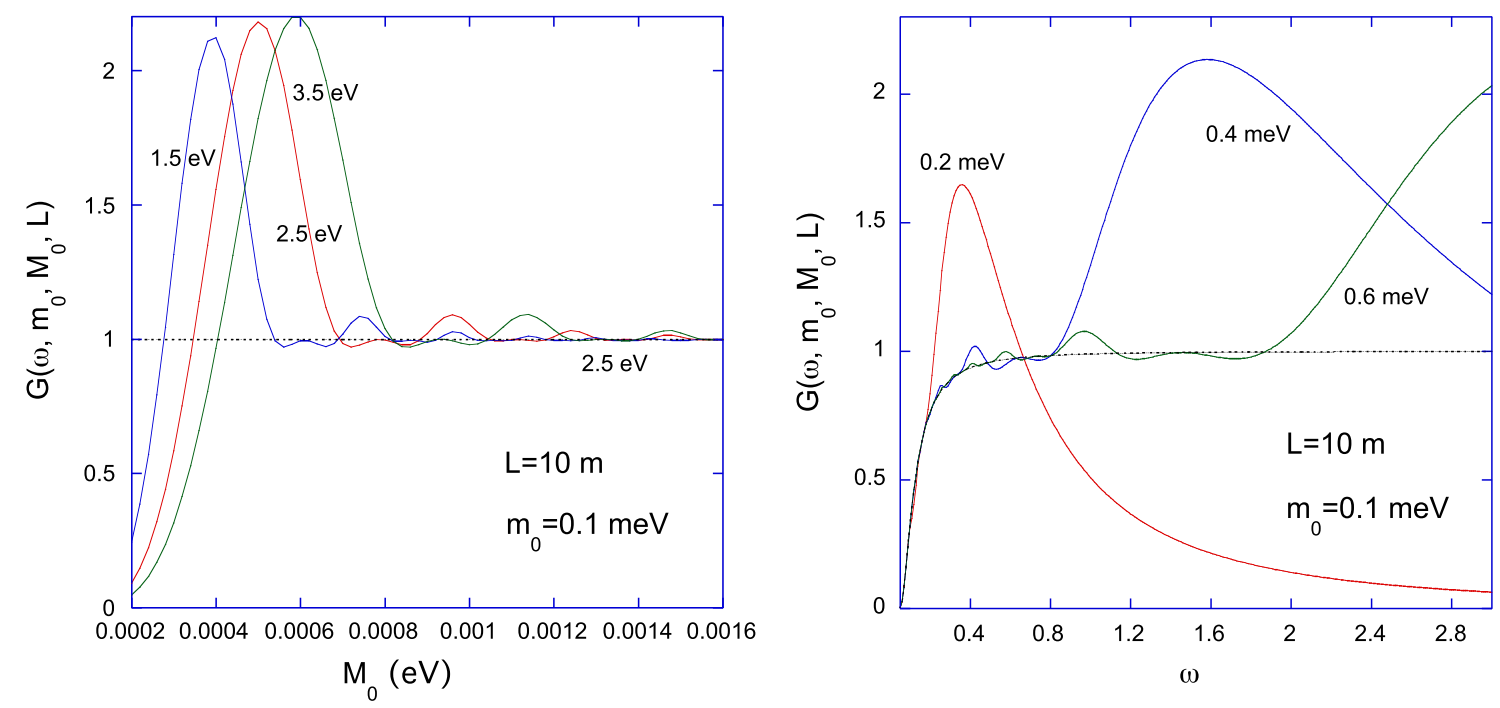

FIG. 1. The function $G$ defined in the expression for the photon regeneration probability [Eq. (3.18)] for fixed $L$ and $m_{0}$. The curves in the left panel are labeled by photon energy $\omega$; in the right panel, by the Lee-Wick ALP mass $M_{0}$.

$$
I(\omega)=\int \frac{d q}{2 \pi} \frac{F(q)^{2} e^{i q L}}{(q+\omega)^{2}-\left(\omega^{2}-m_{0}^{2}\right)-i \epsilon}
$$

and note that $F(q)^{2} e^{i q L}$ has no singularities for any finite complex $q$ and depends only on non-negative powers of $e^{i q L}$. Hence, we can evaluate $q$ the integral along the real axis of the complex $q$ plane by closing the contour at infinity via a semicircle in the upper half-plane. Defining

$$
k_{a}=\sqrt{\omega^{2}-m_{0}^{2}}
$$

the contour just described encloses a pole at $q_{*}=k_{a}-\omega$. By the residue theorem, one thus finds

$$
P=\frac{1}{16} g_{a \gamma \gamma}^{4} B_{0}^{4} L^{4}\left(\frac{\omega}{k_{a}}\right)^{2} F\left(q_{*}\right)^{4} .
$$

This agrees with the expression quoted in Ref. [4], up to a different sign convention for the momentum $q_{*}$.

It is interesting to note that the approach of Ref. [4] involves squaring a $\gamma \leftrightarrow a$ transition probability that is inferred from a classical field amplitude. Using quantum field theory, if one computes the probability of one-intoone $\gamma \rightarrow a$ scattering off a single source (say, the one on the left of the barrier), one finds that the square of this probability also agrees with Eq. (3.16). Though that may provide a convenient shortcut to obtaining the final answer, such a calculation is not the appropriate one: It corresponds to a different physical situation, one in which a measurement is made of the ALP directly, an assumption that cannot apply to the second, Lee-Wick ALP. Our approach does not run into this difficulty.

It is straightforward to include the effect of the Lee-Wick ALP in our approach, by modifying $\left|\frac{i \omega}{2 k_{a}} F\left(q_{*}\right)^{2} e^{i q_{*} L}\right|^{2} \rightarrow\left|\frac{i \omega}{2 k_{a}} F\left(q_{*}\right)^{2} e^{i q_{*} L}-\frac{i \omega}{2 \tilde{k}_{a}} F\left(\tilde{q}_{*}\right)^{2} e^{i \tilde{q}_{*} L}\right|^{2}$,

where $\tilde{q}_{*}$ and $\tilde{k}_{a}$ are defined in the same way as $q_{*}$ and $k_{a}$, with $m_{0}$ replaced by $M_{0}$; the sign difference between terms reflects the difference in the sign of the propagators for the ordinary and Lee-Wick ALPs, as well as a sign flip at each vertex. After including the appropriate dependence on mixing angle in the prefactor, the conversion probability becomes

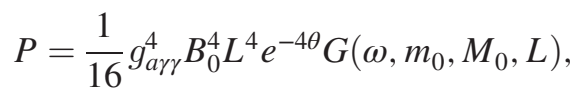

where

$$
\begin{aligned}
& G\left(\omega, m_{0}, M_{0}, L\right) \\
& =\left[\left(\frac{\omega}{k_{a}}\right)^{2} F\left(q_{*}\right)^{4}+\left(\frac{\omega}{\tilde{k}_{a}}\right)^{2} F\left(\tilde{q}_{*}\right)^{4}\right. \\
& \left.\quad-2\left(\frac{\omega}{k_{a}}\right)\left(\frac{\omega}{\tilde{k}_{a}}\right) F\left(q_{*}\right)^{2} F\left(\tilde{q}_{*}\right)^{2} \cos \left[\left(q_{*}-\tilde{q}_{*}\right) L\right]\right] .
\end{aligned}
$$

We plot the function $G\left(\omega, m_{0}, M_{0}, L\right)$ in Fig. 1, as a function of $M_{0}$ (left) and photon energy $\omega$ in the left and right panels, respectively, with other parameters held fixed. For the purpose of illustration, we have fixed $L=10 \mathrm{~m}$ and $m_{0}=0.1 \mathrm{meV}$ in each of these subfigures. We note that $L=10 \mathrm{~m}$ and $\omega=2.5 \mathrm{eV}$ were the benchmark values assumed in Ref. [4]; moreover, current exclusion regions for axions from LSW experiments like OSQAR [15] go up to about $1 \mathrm{meV}$, which motivates the $M_{0}$ range in the left panel in Fig. 1. The line that is at (or asymptotes to) $G=1$ 
corresponds to the case where $M_{0}=\infty$, i.e., where only the normal ALP is present, and is provided as a point of comparison. Note that the separate factor of $e^{-4 \theta}$ in Eq. (3.18) can be computed from Eq. (2.6) and is of $\mathcal{O}(1)$ for values of $m_{0}$ and $M_{0}$ relevant in these figures; for a fixed choice of $m_{0}$ and $M_{0}$, this factor can be absorbed into a redefinition of the coupling $g_{\text {ary }}$. These figures illustrate that the presence of the Lee-Wick ALP can lead to nonnegligible differences in the photon regeneration probability compared to ALP models without the higher-derivative quadratic terms. The right panel of the figure makes clear that this difference might be probed experimentally by performing LSW experiments at different photon energies, where the energy dependence of the conversion probability could become apparent.

A number of comments are in order: (i) One might object that the sensitivities of current LSW experiments are superseded by other bounds on light particles, particularly from searches for solar axions (see the bound from the CAST experiment [16] in the exclusion plot in Ref. [5]). While this is true, the present result is of interest due to the substantial room for improvement in the sensitivity anticipated for future LSW experiments, corresponding to values of $g_{A \gamma \gamma}$ that are more than 4 orders of magnitude smaller than those accessible by LSW experiments today. This is illustrated by the projected exclusion region for the proposed ALPS-II [17], ALPS-III [18], and STAX2 [19] experiments in Fig. 6 of Ref. [3]. The basic qualitative conclusion of the present work, that it may be useful to look at the dependence of the conversion probability on photon energy to search for effects like those shown in the right panel in Fig. 1, is potentially of value. (ii) Those who have worked with Lee-Wick theories in other contexts may have anticipated a suppression of the squared amplitude due to the cancellation between the ordinary and the Lee-Wick particle propagators (the effect that leads to better convergence of loop diagrams in the LeeWick standard model). However, this is not the case here, since each term in the amplitude is a finite integral in $q$ that is dominated by $q_{*}$, as is clear from Eq. (3.19), and $q_{*} \ll m_{0}$. [Note that when $m_{0} \ll \omega$, as we have been assuming, $q_{*} \approx m_{0}^{2} /(2 \omega) \ll m_{0}$.] Hence, the ultraviolet behavior of the integrand is not determining the final result. (iii) We presented the form of the photon-ALP vertex in the case where the ALP is scalar rather than pseudoscalar in Sec. I. In this case, the amplitude given in Eq. (3.4) would be modified by the replacement

$\epsilon^{\mu \nu \alpha \beta} \epsilon^{\rho \sigma \kappa \eta} \rightarrow\left(g^{\mu \alpha} g^{\nu \beta}-g^{\nu \alpha} g^{\mu \beta}\right)\left(g^{\rho \kappa} g^{\sigma \eta}-g^{\sigma \kappa} g^{\rho \eta}\right)$.

The subsequent derivation is the same as the one described in the case of a pseudoscalar ALP, and the final result turns out to be identical to the one given in Eqs. (3.18) and (3.19), with only the notational replacement $g_{a \gamma \gamma} \rightarrow g_{s \gamma \gamma}$. (iv) The LeeWick ALP could have a width $\Gamma$ that is larger in magnitude than the ordinary ALP, depending on the model. When a width is included, the exact Lee-Wick propagator has a pair of complex conjugate poles and a cut. In the narrow width approximation, one of these poles and the cut piece cancel, so that a single pole remains, with $M^{2}$ replaced by $M^{2}+i M|\Gamma|$; this expression reflects the fact that Lee-Wick particles have $\Gamma<0$. Notice that the width moves the location of propagator poles in a direction opposite that given by the $i \epsilon$ prescription. The Lee-Wick prescription [6] requires that an integration contour that is initially on the real axis and given by the Feynman prescription is deformed as the width is turned on, so that the poles remain in the same relative location, either above or below the contour, without crossing it. The end result in the present calculation would be to replace $M^{2}$ by $M^{2}+$ $i M|\Gamma|$ in Eq. (3.17) and modify Eqs. (3.19) accordingly. We have done this exercise and checked that including a width of the order of $0.01 M_{0}-0.1 M_{0}$ in our previous calculation does not change the results shown in Fig. 1 qualitatively.

\section{CONCLUSIONS}

We have considered the consequences of higher-derivative quadratic terms in models with ALPs. Such higher-derivative terms have been of interest in extensions of the standard model intended to address the hierarchy problem [7], as well as in attempts to formulate renormalizable theories of quantum gravity [20]. Their generic presence is well motivated, and the mass scales associated with these terms are free parameters. Higher-derivative quadratic terms in ALP models give rise to a second ALP, a Lee-Wick partner [6]. Their appropriate treatment in quantum field theory is well known $[6,8]$ and requires that they be excluded from the spectrum of asymptotic scattering states. Nevertheless, if the Lee-Wick ALP is not decoupled from the relevant low-energy effective theory, it can have observable consequences. We have shown this in the context of LSW experiments, where the ordinary and Lee-Wick ALPs have similar interaction vertices with photons (up to a sign) and can both contribute to the photon regeneration amplitude. Our result shows a distinctive dependence of the regeneration probability on incident photon energy. With substantial improvements in the sensitivity of LSW experiments anticipated [17-19], such an effect might be relevant experimentally in distinguishing the possibility considered here from more conventional ALP scenarios.

\section{ACKNOWLEDGMENTS}

We are grateful to Josh Erlich and Marc Sher for useful comments. We thank the National Science Foundation for support under Grant No. PHY-1819575. 
[1] S. Weinberg, A New Light Boson?, Phys. Rev. Lett. 40, 223 (1978); F. Wilczek, Problem of Strong $P$ and $T$ Invariance in the Presence of Instantons, Phys. Rev. Lett. 40, 279 (1978).

[2] R. D. Peccei and H. R. Quinn, $C P$ Conservation in the Presence of Instantons, Phys. Rev. Lett. 38, 1440 (1977); Constraints Imposed by $C P$ conservation in the presence of instantons, Phys. Rev. D 16, 1791 (1977).

[3] I. G. Irastorza and J. Redondo, New experimental approaches in the search for axion-like particles, Prog. Part. Nucl. Phys. 102, 89 (2018).

[4] K. Van Bibber, N. R. Dagdeviren, S. E. Koonin, A. Kerman, and H. N. Nelson, Proposed Experiment to Produce and Detect Light Pseudoscalars, Phys. Rev. Lett. 59, 759 (1987).

[5] See Axions and Other Similar Particles in P. A. Zyla et al. (Particle Data Group), Review of particle physics, Prog. Theor. Exp. Phys. 2020, 083C01 (2020).

[6] T. D. Lee and G. C. Wick, Negative metric and the unitarity of the S matrix, Nucl. Phys. B9, 209 (1969); Finite theory of quantum electrodynamics, Phys. Rev. D 2, 1033 (1970).

[7] B. Grinstein, D. O'Connell, and M. B. Wise, The Lee-Wick standard model, Phys. Rev. D 77, 025012 (2008).

[8] R. E. Cutkosky, P. V. Landshoff, D. I. Olive, and J. C. Polkinghorne, A non-analytic S matrix, Nucl. Phys. B12, 281 (1969).

[9] See, for example, G. R. Farrar, Experiments to find or exclude a longlived, light gluino, Phys. Rev. D 51, 3904 (1995); L. Clavelli, Possible indication of a light gluino, Phys. Rev. D 46, 2112 (1992).

[10] See, for example, E. J. Chun and H. B. Kim, Axino light dark matter and neutrino masses with R-parity violation,
J. High Energy Phys. 10 (2006) 082; K. Choi, E. J. Chun, and J. S. Lee, Proton decay with a light gravitino or axino, Phys. Rev. D 55, R3924 (1997).

[11] H. Georgi, Lie algebras in particle physics, Front. Phys. 54, 1 (1999).

[12] C. D. Carone and R. Primulando, Constraints on the LeeWick Higgs sector, Phys. Rev. D 80, 055020 (2009).

[13] B. Grinstein, D. O'Connell, and M. B. Wise, Causality as an emergent macroscopic phenomenon: The Lee-Wick $\mathrm{O}(\mathrm{N})$ model, Phys. Rev. D 79, 105019 (2009).

[14] M. E. Peskin and D. V. Schroeder, An Introduction to Quantum Field Theory (Westview Press, Boulder, CO, 1995).

[15] R. Ballou et al. (OSQAR Collaboration), New exclusion limits on scalar and pseudoscalar axionlike particles from light shining through a wall, Phys. Rev. D 92, 092002 (2015).

[16] V. Anastassopoulos et al. (CAST Collaboration), New CAST limit on the axion-photon interaction, Nat. Phys. 13, 584 (2017).

[17] R. Bähre, B. Döbrich, J. Dreyling-Eschweiler, S. Ghazaryan, R. Hodajerdi, D. Horns, F. Januschek, E. A. Knabbe, A. Lindner, D. Notz et al. Any light particle search II-Technical design report, J. Instrum. 8, T09001 (2013).

[18] A. Lindner, Physics Beyond Colliders Annual Workshop (CERN, 2017), https://indico.cern.ch/event/644287/.

[19] L. Capparelli, G. Cavoto, J. Ferretti, F. Giazotto, A. D. Polosa, and P. Spagnolo, Axion-like particle searches with sub-THz photons, Phys. Dark Universe 12, 37 (2016).

[20] L. Modesto, Super-renormalizable or finite Lee-Wick quantum gravity, Nucl. Phys. B909, 584 (2016). 